\title{
Trauma-Informed Neurofeedback and Expressive Arts Therapy: Wilson Disease Case Report
}

\author{
Kian Yong Koo, M.S. ${ }^{1}$, Azizah Binti Abdullah, Ph.D. ${ }^{2}$, and Jun Ming Loh, \\ B.S. ${ }^{3},{ }^{1}$ School of Education, UUM College of Arts and Sciences, Universiti \\ Utara Malaysia, ${ }^{2}$ School of Education, UUM College of Arts and Sciences, \\ Universiti Utara Malaysia, ${ }^{3} \mathrm{New}$ Mind Brain Health Centre, Kuala Lumpur, \\ Email: hirokoo09@gmail.com, $\quad$ zyzah@uum.edu.my, \\ newmindcentre@gmail.com
}

A case report on enhancing self-regulation skills through QEEGguided neurofeedback was conducted for a subject who has been diagnosed with Wilson Disease (WD). The trauma-informed neurofeedback training protocols were developed to improve the client's self-regulation skills in order to address his psychological symptoms and cognitive impairment. The protocols were developed based on the findings of the prior empirical research as well as the QEEG brain mapping assessment of the client. In this case report, the utilisation of expressive art therapy application was highlighted in facilitating the therapeutic process for the clients having difficulty in verbal communication. The QEEG brain mapping assessment result pre-and-post intervention were compared to determine the progress of the client's brainwave activity pattern. Meanwhile, the self-report diary of the client's parent was used to follow up his conditions. The outcome indicated that the developed protocols of trauma-informed neurofeedback training and expressive art application were helpful in improving the self-regulation skills of the client.

Key words: QEEG-guided neurofeedback training, Trauma-informed protocols, Expressive art therapy, New Mind Card, Wilson disease, Self-regulation, Brain mapping assessment 


\subsection{INTRODUCTION}

In this era of advanced healthcare technologies, the complementary and alternative treatments are widely accepted and accessible by the society [National Institutes of Health (NIH), 2007]. Legarda and her team (Legarda, McMahon, Othmer \& Othmer, 2011) highlighted that there is a rising in the consumer spending for seeking alternative treatments and therapies, especially the clinical neurofeedback training for chronic neurological conditions. In neurofeedback training, the targeted bandwidth brainwave would be up-regulated or down-regulated according to the individuals' condition (Marzbani, Marateb \& Mansourian, 2016). The targeted bandwidth brainwave was chosen to train primarily based on its impacts towards the individual. Table 1 summarises the distinct bandwidth brainwaves with their respective characteristic.

Table 1 Types of Brainwaves

\begin{tabular}{|c|c|c|}
\hline $\begin{array}{l}\text { Common Brainwave } \\
\text { Frequency }\end{array}$ & $\begin{array}{l}\text { Frequency } \\
\text { Range }(\mathrm{Hz})\end{array}$ & General Characteristics \\
\hline Delta & $1-4$ & $\begin{array}{l}\text { Sleep, repair, complex problem solving, } \\
\text { unawareness, deep-unconsciousness }\end{array}$ \\
\hline Theta & $4-8$ & $\begin{array}{l}\text { Creativity, insight, deep states, unconsciousness, } \\
\text { optimal meditative state, depressive, anxiety, } \\
\text { distractibility }\end{array}$ \\
\hline Alpha & $8-13$ & $\begin{array}{l}\text { Alertness and peacefulness, readiness, meditation, } \\
\text { deeply-relaxed, recalling, optimal cognitive } \\
\text { performance }\end{array}$ \\
\hline $\begin{array}{l}\text { SMR (sensorimotor } \\
\text { rhythm) }\end{array}$ & $13-15$ & Mental alertness, physical relaxation \\
\hline Beta & $15-20$ & $\begin{array}{l}\text { Thinking, focusing, sustained attention, tension, } \\
\text { alertness, excitement }\end{array}$ \\
\hline Hi-Beta & $20-32$ & Intensity, hyperalertness, anxiety \\
\hline Gamma & 32 and above & $\begin{array}{l}\text { Learning, cognitive processing, problem solving } \\
\text { tasks, mental sharpness, brain activity, organise the } \\
\text { brain }\end{array}$ \\
\hline
\end{tabular}

In recent years, the research on Quantitative Electroencephalography (QEEG)-guided neurofeedback training has attracted the interests of researchers. In QEEG-guided neurofeedback training, the training protocols are designed accordingly to the QEEG brain mapping result of the individual. For this, the QEEG brain mapping was found to be highly validated and a reliable tool to access and localise brain activity and brain function of the individuals (Linardakis \& Pardell, 2018; Marzbani et al., 2016). Therefore, QEEG-guided neurofeedback has catered the clinical needs in customising the training protocols for different individuals who reported similar symptoms due to the variety in manifestation of an underlying electrophysiological pattern (Orndorff-Plunkett, 2017; Rogala et al., 2016). 
Despite the potential clinical efficacy of neurofeedback training, the non-responder's concerns are the issue which should be attended to. Research study has suggested that work on mood directly was more effective than work on the brain area which is associated with emotion (Kadosh \& Staunton, 2019). This is because psychological variables are indeed influencing the neurofeedback training and self-regulation outcome. In order to ensure the positive neurofeedback training outcome, it is crucial to deal with the psychological variables during the training process (Kadosh \& Staunton, 2019). Therefore, the integration of an expressive arts application with a neurofeedback training session was speculated to foster a better cognitive rehabilitation process and psychological well-being for the client.

An expressive arts application enables individuals who experienced traumatic events to heal through the expression and creation of arts (Riley, 2001). It was developed by Natalie Rogers. She integrated the philosophy of a person-centred approach from Carl Rogers and utilised them (i.e., congruence, unconditional positive regard and empathy) to create a psychological safety environment (Chiang, Reid-Varley \& Fan, 2019). This provides an opportunity for individuals to express their inner self or unconscious minds through techniques such as picking up an art therapy card, drawings or painting (Malchiodi, 2020). Therefore, it was believed that the expressive arts therapy will facilitate a more efficient treatment session which involve the mutual communication between the client and the therapist.

In this report, the authors have presented a case report of developing trauma-informed protocols in QEEG-guided neurofeedback training as well as expressive art therapy application to improve the self-regulation skill in managing the psychological symptoms and cognitive impairment of the client who has been diagnosed with Wilson Disease (WD). The client has suffered from brain injury symptoms after the WD and he engaged in the neurofeedback training as a cognitive rehabilitation method to work on his condition. Previous study acknowledges the contribution of neurofeedback training in cognitive improvement for braininjured populations (Ali, Viczko, \& Smart, 2020). However, one significant challenge for cognitive rehabilitation is about the lack of theoretical consensus. Thus, it is crucial for this study to develop an intervention protocol which fosters a clearer operational definition, precise therapeutic targets, as well as anticipated outcomes. Meanwhile, this report also emphasised the facilitator role of expressive arts application, mainly the newly invented New Mind Card, to develop an integrated trauma-informed protocol in maximising the treatment efficiency.

\subsection{CASE DESCRIPTION}

\subsection{Case Overview}

$\mathrm{K}$ is the pseudonym used to address the client in this present case report. $\mathrm{K}$ was born in 1997 without any significant health conditions reported upon his birth. However, K started to display various ailments including vomiting blood and momentary blackouts in November 2015. Later, it was found that K's liver and gallstone had significantly defected and scarred. He had to 
receive treatment to remove his gallbladder upon the diagnosis at one of the local private hospitals. It was reported that the gastroenterologist suspected $\mathrm{K}$ may have been affected by WD. K was later referred to one of the local government hospitals, where he was diagnosed with WD in February 2016 by the doctors.

WD has significantly affected K's life. He was forced to postpone his enrolment twice to an abroad university between 2016-2017. The WD symptoms that K displayed were summarised in Table 2.1 and Table 2.2. The symptoms mentioned have significantly limited the physical and cognitive functioning level of $\mathrm{K}$ which ultimately resulted in psychological issues.

$\mathrm{K}$ has dedicated his endeavour in receiving various kinds of intervention to combat the psychological symptoms that arise from his condition. Since his diagnosis, K has tried different interventions including specialist rehabilitation, traditional treatment, and speech therapy. In 2018, K started receiving clinical neurofeedback training as an intervention. Due to K's difficulty in expression, the neurofeedback training session had added with the expressive arts application to facilitate interaction with him.

\subsection{Clinical Presentation}

WD is a genetic and neurological disorder that is rooted from the abnormal accumulation of copper in various organs, including the brain, due to the distorted metabolism of the individual [National Organization for Rare Disorders (NORD), 2019]. Table 2.1 summarised the presenting neurological symptoms of $\mathrm{K}$ prior to the interventions provided.

Table 2 The Presenting Neurological Symptoms of K

\begin{tabular}{|c|l|}
\hline $\begin{array}{c}\text { Neurological } \\
\text { Symptoms }\end{array}$ & \multicolumn{1}{|c|}{ Descriptions } \\
\hline Dysarthria & $\begin{array}{l}\text { Difficulty in producing speech which results in the slurred speech of K } \\
\text { in expressing his thoughts. }\end{array}$ \\
\hline Dysphagia & $\begin{array}{l}\text { Difficulty in swallowing and thus result in the uncontrollable } \\
\text { salivation (drooling) and inability to swallow the food. }\end{array}$ \\
\hline Dystonia & $\begin{array}{l}\text { Clawed parts on } 3^{\text {rd }} \text { to } 5^{\text {th }} \text { fingers on both hands and } 3^{\text {rd }} \text { to } 5^{\text {th }} \text { toes on } \\
\text { feet which limits the motor function and controllability on the limbs of } \\
\text { K. }\end{array}$ \\
\hline
\end{tabular}

In addition, $\mathrm{K}$ also displayed some psychological symptoms, which displayed in Table 2.2. In concordance to the highlights of European Association for Study of Liver (2012), the presenting psychological symptoms was mainly due to the cognitive impairment that resulted from abnormal high level of copper accumulated in the brain and it leads to brain injury issues. 
International Journal of Innovation, Creativity and Change. www.ijicc.net

Volume 15, Issue 4, 2021

Table 3 The Presenting Psychological Symptoms of K

\begin{tabular}{|c|c|}
\hline $\begin{array}{l}\text { Psychological } \\
\text { Symptoms }\end{array}$ & Descriptions \\
\hline $\begin{array}{l}\text { Obsessive } \\
\text { thoughts }\end{array}$ & $\begin{array}{l}\text { a. Craving for food frequently during the night. } \\
\text { b. Asking for a face towel to be tied around the mouth to wipe } \\
\text { off the saliva about four times an hour. } \\
\text { c. Demanding the loud volume of television or phone despite not } \\
\text { using the device. } \\
\text { d. Mentally attaching to certain items which must be brought } \\
\text { along wherever K goes. } \\
\text { e. } \\
\text { walk. Requesting to leave the house in the middle of the night for a } \\
\text { f. } \\
\text { g. } \\
\text { Touching the phone screen without any goal. } \\
\text { i.Bring him the drinks; } \\
\text { ii.accompany him to the toilet; } \\
\text { iii.cover him with a blanket while sleeping. }\end{array}$ \\
\hline $\begin{array}{l}\text { Compulsive } \\
\text { behaviours }\end{array}$ & $\begin{array}{l}\text { K would behave compulsively (i.e., wailing, bawling and groaning) if } \\
\text { he failed to carry out the obsessive thoughts aforementioned. }\end{array}$ \\
\hline Sleep disturbance & $\begin{array}{l}\text { K encountered difficulty in falling asleep and having refreshing } \\
\text { sleep. }\end{array}$ \\
\hline
\end{tabular}

The intense anxiety that $\mathrm{K}$ experienced is consistent with the prior finding whereby the uncontrollable and recurrent thoughts and behaviours will cause anxiety in the individual, and this anxious feeling will be more intense when the obsessive thoughts were inhibited (Goodwin, 2015). Moreover, it was also reported that $\mathrm{K}$ tends to ruminate about his symptoms and its impacts whenever he felt anxious. This is in line with the most empirical supported definition of rumination whereby it is the focused attention on one's distress, and on its possible causes and consequences (Nolen-Hoeksema, 2000). Pertaining to the notion of the previous study, ruminative thoughts were found to be a common response towards the anxiety feelings (NolenHoeksema, 2000). In K, the ruminative thoughts were mainly manifested in his concern about his condition that would never allow him to do things as before.

\subsection{Interventions: Clinical QEEG-Guided Neurofeedback Training Integrated with Expressive Art Therapy Protocols}

QEEG guided neurofeedback is the intervention implemented in this present case report. The pre-intervention QEEG assessment was implemented to obtain the brainwave activities patterns of $\mathrm{K}$, and the result was used in generating the neurofeedback training protocols (trauma-informed clinical neurofeedback training protocols) and expressive art therapy protocols (trauma-informed expressive art therapy using New Mind Card technique). A post- 
intervention QEEG assessment was conducted to track the progress on the brainwave activities patterns of $\mathrm{K}$ after attending 74 sessions of neurofeedback training. Throughout the sessions, New Mind Card was used to address the psychological variables in order to maximise the efficiency of the treatment process. In addition, the self-reported diary log of K's father, was accessed to track on K's conditions beyond the termination of the therapeutic process.

\subsubsection{Trauma-informed Expressive Art Therapy Application}

In this study, the expressive art therapy was utilised to help $\mathrm{K}$ expresses his emotions and feelings. Due to K's difficulty in verbal expression, the New Mind Card was used to assist him to break the silence in nonverbal way. This idea of "tell without talking" is aligned with the idea of Dr. Cathy Malchiodi, (2020). The New Mind Card is designed by the researcher, Koo Kian Yong, in accordance to impairment domains of complex trauma on children as highlighted by Spinazzola et al (2005). It is created based on impairment domains after a complex trauma event (see appendix: New Mind Card). All these cards are created to provide a non-verbal way in expressing the impact of potential complex traumas in one's life. The technique of using the New Mind Card is parallel with the trauma intervention model of Herman's and Bessel's framework of recovery, which help the client to gain a sense of safety, establishing vision for the future, self-regulation, dealing with trauma response pattern and paying attention to the embodied experience (Malchiodi, 2020).

\subsubsection{Trauma-informed QEEG Guided Neurofeedback Training Protocols}

There were four different sets of protocol that have been developed for K. During March 2018 to mid-July 2019, K has undergone 51 sessions of QEEG guided neurofeedback training under the protocol of FZ+SMR and CZ+SMR; and 45 sessions under the protocol of F7+SMR/alpha and $\mathrm{F} 8+\mathrm{SMR} /$ alpha. In between, father of $\mathrm{K}$ requested to switch his protocol and undergo nine sessions of T3+SMR and C3+beta; and five sessions of T4+delta and P4+delta to improve his calming thoughts and sleep. Figure 2.1 demonstrates the EEG electrodes placement under 1020 international system for $\mathrm{K}$.

In this study, the bottom approach philosophy was adopted in generating training protocol sequences. The bottom-up approach works on the brain stem to regulate the sensation and stress arousal, then gradually works on the limbic system to improve the emotional state and lastly works on the cortex to enhance the cognitive functions (Ogden, Minton \& Pain, 2020). Thus, the initial phrase of the training starts with the mid brain or brainstem related area $(\mathrm{Cz}, \mathrm{C} 3$ and $\mathrm{P} 4)$, then gradually moving toward the limbic system area of the brain (T3 and T4) and lastly the language expression as well as cognition related area of the brain (F7 and F8). However, the bottom-up approach in this study provides flexibility throughout the treatment course depending on the client's needs and status. For example, when the client faces difficulty in sleeping, the protocol might be adjusted to improve the sleep concern. This is aligned with the philosophy of trauma-informed expressive art therapy whereby if accessing the brain stem too 
quickly, it may provoke dissociation or worsen the symptoms (Malchiodi, 2020). Thus, it is important to monitor the individual's pace, unique brainwaves pattern and the response to the training throughout the intervention.

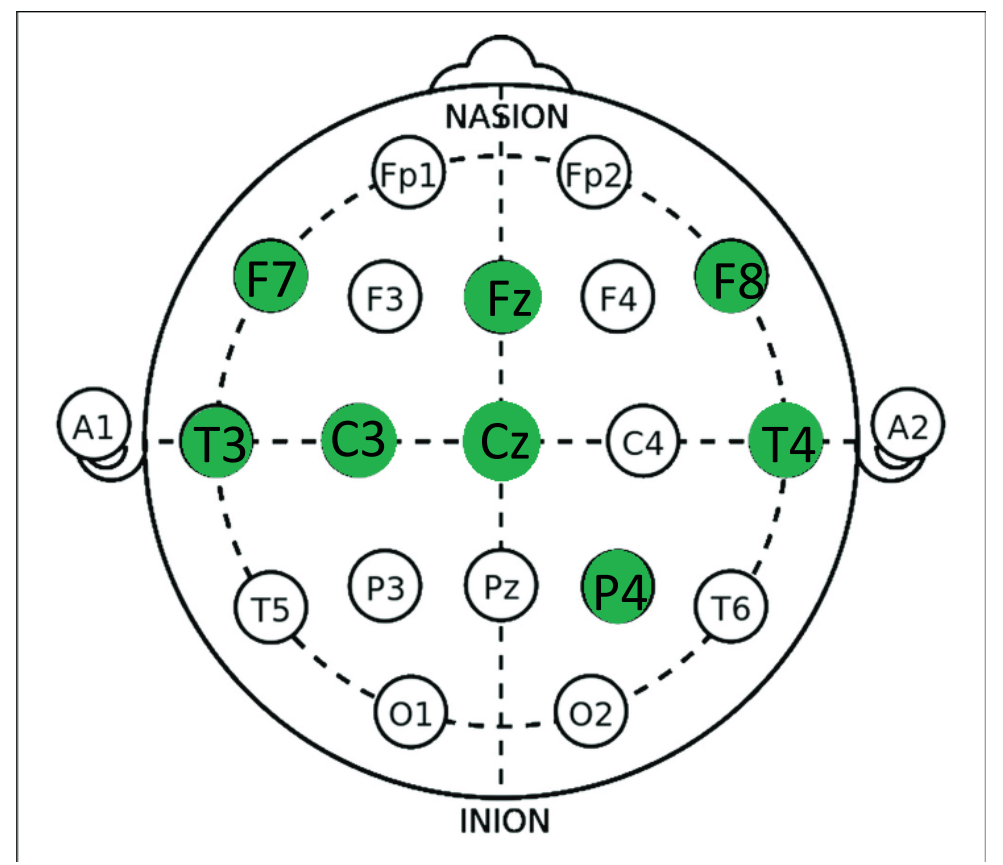

Figure 2.1. 10-20 International System Placement for EEG Electrodes in K's case

\subsubsection{Rewarding SMR on Fz \& $\mathrm{Cz}$}

This protocol was designed to train the cortex associated area, particularly the anterior cingulate gyrus associated area (i.e., Fz and $\mathrm{Cz}$ ) which found to be vital for the higher-ordering cognitive functions (Wang et al., 2020). This protocol was implemented on Fz to improve the inhibitory behaviours and executive functioning as well as $\mathrm{Cz}$ to improve motor functions of $\mathrm{K}$. Meanwhile, the design of this particular training protocol was mainly based on the preintervention QEEG brain mapping assessment as displayed in Figure 3.1, which indicated the dominance of the slow bandwidth brainwaves (i.e., delta and alpha brainwaves).

Pertaining to K's condition, it was speculated that his presenting symptoms were manipulated by the deficit in executive functioning. In this case, the frontal lobe regions have found to be important for one's executive functioning level due to its roles in high-ordering cognitive processing, mood regulation, motor function, and language skills (Heatherton, 2011). Furthermore, researchers found that the deficit in executive functioning tends to result in poor impulse inhibition and emotion control (King, Fleming, Monahan \& Catalano, 2012). These findings were in accordance with the symptoms that manifested in K. Henceforth, the present neurofeedback training protocol was implemented to improve his executive functioning level. 
Prior research has highlighted that elevating SMR brainwave was found to enhance the cognitive performance such as memory consolidation and working memory performance (Zoefel, Huster, Herrmann, 2011). In the meantime, the alpha brainwave was down-regulated to induce the cortical activation of the particular training brain region (Wang \& Hsieh, 2013). Therefore, the present training protocol was designed to reward SMR and inhibit the slow bandwidth brainwaves to improve the executive functioning, working memory and attentional focus of $\mathrm{K}$.

Fz protocol training is also parallel with the concept of neurodevelopment in which the executive function is related with the cortex (Malchiodi, 2020) and thus, the usage of the New Mind Card during this stage of protocol training was mainly used for skill enhancement and self-esteem exploration. For instance, the therapist asked $\mathrm{K}$ to explore which domain of impairment he would like to improve first by picking up few cards and he then expressed his feelings by answering with a text message. Meanwhile, the $\mathrm{Cz}$ training is aligned with the concept of neurodevelopment which highlighted motor skill as well as coordination, which are related with the midbrain area (Malchiodi, 2020). Therefore, physically oriented activities such as encouraging $\mathrm{K}$ to type a text message via the phone was implemented to improve his motor skill and coordination.

\subsubsection{Rewarding SMR and alpha on F7 \& F8}

This training protocol was developed and implemented on the left and right frontal region of K's brain. This particular training protocol was developed to reduce the anxious feeling, regressive cognitive and behavioural patterns of $\mathrm{K}$. Meanwhile, activation of the left frontal lobe region (i.e., known as F7 in 10-20 international system) was speculated to be helpful for the verbal expression of $\mathrm{K}$, whereby it is the Broca's area associated region.

In prior neuro-imaging study, the frontal lobe was found to significantly link to one's ability to regulate the negative emotions (Ochsner, Silvers \& Buhle, 2012). Activation of the frontal lobe, especially the left frontal lobe region of the brain is associated with a low level of anxiety and low re-experiencing of intrusive trauma-related memories (Lu et al., 2017). Furthermore, research has found that activation level of the frontal part of the brain is negatively associated with emotion dysregulation and severity of anxiety (Ball et al., 2013). Therefore, it was speculated that through improving the SMR brainwave on the frontal lobes, the regulation of negative emotions, particularly the ruminative anxious feelings in $\mathrm{K}$ would be improved as well.

Meanwhile, alpha-training in neurofeedback training was found to significantly reduces one's anxious feelings with lasting effects (Moradi et al. 2011). Thus, alpha brainwave was reinforced at the same time with the SMR brainwave on F7 and F8 throughout the neurofeedback training sessions to alleviate K's anxiety as well as ruminative thoughts. 
Moreover, this protocol was in line with the concept of neurodevelopment whereby the affect regulation is related with the limbic system (Malchiodi, 2020). The aforementioned roles of both left and right frontal parts in regulating emotion is highly consistent with the functions of limbic system. In the prior papers, frontal lobes also have been granted as the anterior limbic network due to its vital role in affect regulation (Grodd, Kumar, Schuz, Lindig \& Scheffler, 2020). Thus, the New Mind Card was used for $\mathrm{K}$ to express creatively during this stage of training (e.g., let him pick a card and express freely about how he feels about the card and what is the coping skill that he can work on it by letting him express in text message form).

\subsubsection{Rewarding SMR on T3 and rewarding beta on $\mathrm{C3}$}

This protocol was developed to promote psychological wellness of K. This present protocol was focusing on reducing hyper-activation of the left temporal lobe, which is known as T3 in the 10-20 international system. Ferdek and his colleagues (van Rijn \& Wyczesany, 2016) have found that the hyperactivation on the left temporal lobe is crucial for the ruminative tendencies. Torres et al. (2016) has highlighted the positive association between ruminative tendencies and generalised anxiety disorder. Therefore, it was speculated that the anxious feeling that experienced by $\mathrm{K}$ was rooted in his ruminative thoughts' pattern or vice versa (due to the dominance of the hi-beta brainwave) on his left temporal lobe, T3 as shown in Figure 3.1. Therefore, by reducing the activation level and reinforcing the SMR brainwave, it was believed to alleviate the anxious feelings and the obsessive thoughts of $\mathrm{K}$.

Meanwhile, the reinforcement of beta brainwave on C3 was developed to improve K's mindfulness skills in order to combat his ruminative thought pattern. It was highlighted that the mindfulness skills are able to reduce the uncontrollable ruminative cycles which result in the re-experiencing intrusive memory of the traumatic experiences (Fenster, Lebois, Ressler \& Suh, 2018). For this, Gao et al. (2016) have found that there was a significant association between the beta brainwave and mindfulness state. In addition, the sensorimotor cortex was granted as the affective component of physical condition due to its role in manipulating emotions such as calm, agitation, anxiety and fear (Jensen, Grierson, Tracy-Smith, Bacigalupi \& Othmer, 2008). Therefore, the enhancement of mindfulness skills via rewarding the low beta brainwave on the left sensorimotor cortex, C3 was believed to reduce the ruminative thoughts and anxious feeling of $\mathrm{K}$.

This set of neurofeedback protocol, especially the T3 training is actually parallel with the concept of neurodevelopment whereby the affect regulation is related with the limbic system (Malchiodi, 2020). For this, the left temporal lobe was regarded as a part of the limbic system as it contains the hippocampus which is important for the formation and storage of the longterm memory (Jo et al., 2019). During this stage, K was encouraged to express his emotions and feelings, which could be related to his past unpleasant experiences by utilising the New Mind Card. 


\subsubsection{Rewarding delta on $\mathrm{T} 4$ and $\mathrm{P} 4$}

This protocol was developed to improve K's sleep issue. Delta training was found to improve sleep quality through increasing the sleep spindle density and alleviating the sleep onset insomnia (Arns \& Kenemans, 2014). In the 10-20 international system, the right temporal lobe also known as T4, was found to be significantly associated with traumatic stress and an anxiety feeling (Lu et al., 2017). The sleep issue reported by $\mathrm{K}$ was linked to his anxious feeling, which was displayed by the high activation level of hi-beta brainwave when closing his eyes. Sherin and Nemeroff (2011) also highlighted that the trauma-related experiences will cause high neural activity in the temporal lobe which ultimately provokes the hypervigilance state of the individual. As a result, the individual tends to be in an anxious state consistently and encounters poor sleep quality (Ramsawh, Stein, Belik, Jacobi \& Sarreenm 2009). The pioneer researchers in the neurofeedback field (Putman, Othmer, Othmer \& Pollock, 2005) have highlighted that the neurofeedback training conducted on the temporal lobes (i.e., T3, T4, T5 \& T6 site), which is effective in promoting general calmness and generalisation of moods.

Besides, prior study in neurofeedback training has highlighted that the training conducted on the right parietal lobe; P4 was efficient in improving the sleep quality of individuals with subclinical anxiety-related symptoms (Gomes, Ducos, Akiba \& Dias, 2016). This is primarily due to the vital role of $\mathrm{P} 4$ in anxiety resilience, whereby the right parietal lobe was found to be significant in responding and attenuating stress, which pertains to its functions as the sensory input processor (Grieder, Homan, Federspiel, Kiefer \& Hasler, 2020). Meanwhile, P4 training was commonly implemented in tackling the sleep issues, such as the sleep difficulty that resulted from the anxiety symptoms of individuals (Jensen et al., 2008). Therefore, the present neurofeedback training protocol was adopted and implemented to enhance the sleep quality of $\mathrm{K}$.

This training protocol was aligned with the concept of neurodevelopment in which the stress responses are related with the brainstem (Malchiodi, 2020). Pertaining to the aforementioned significant role of P4, particularly its activation level, would tend to alter the brain circuit network that are involved in vigilance provoking network (Grieder et al., 2020). Henceforth, an expressive art therapy application was implemented alongside the present neurofeedback training protocol to explore the potential causes of K's anxiety, and provide an alternative for $\mathrm{K}$ to express. Therefore, the New Mind Card was used to explore the experiences of social connection and approval that he longs for, which postulated to be the root of his feeling of insecurity and anxiety. 
International Journal of Innovation, Creativity and Change. www.ijicc.net

Volume 15, Issue 4, 2021

\section{Summary of the neurofeedback training integrated with expressive art protocol}

\begin{tabular}{|l|l|l|}
\hline $\begin{array}{l}\text { Neurofeedback Training } \\
\text { Location }\end{array}$ & $\begin{array}{l}\text { Related Brain } \\
\text { Area }\end{array}$ & $\begin{array}{l}\text { Proposed Expressive Art Therapy } \\
\text { Application }\end{array}$ \\
\hline Fz & Cortex & $\begin{array}{l}\text { Skill enhancement and self-esteem } \\
\text { exploration }\end{array}$ \\
\hline Cz & Midbrain & $\begin{array}{l}\text { Encouraging physically oriented } \\
\text { activities }\end{array}$ \\
\hline T3 or T4 or C3 & Limbic system & Express emotions and feelings creatively \\
\hline F7 or F8 & Limbic system & Express emotions and feelings creatively \\
\hline P4 & Brainstem & $\begin{array}{l}\text { Explore the experiences of social } \\
\text { connection and approval }\end{array}$ \\
\hline
\end{tabular}

\subsection{RESULTS}

The following displays the pre- and post- intervention QEEG brain mapping assessment result of K. A comparison on the pre- and post-intervention QEEG brain mapping assessment was made in order to determine the improvement of K's brainwave activity pattern after attending 74 sessions of neurofeedback training.

\subsection{The Pre-Intervention of QEEG Brain Mapping Assessment}

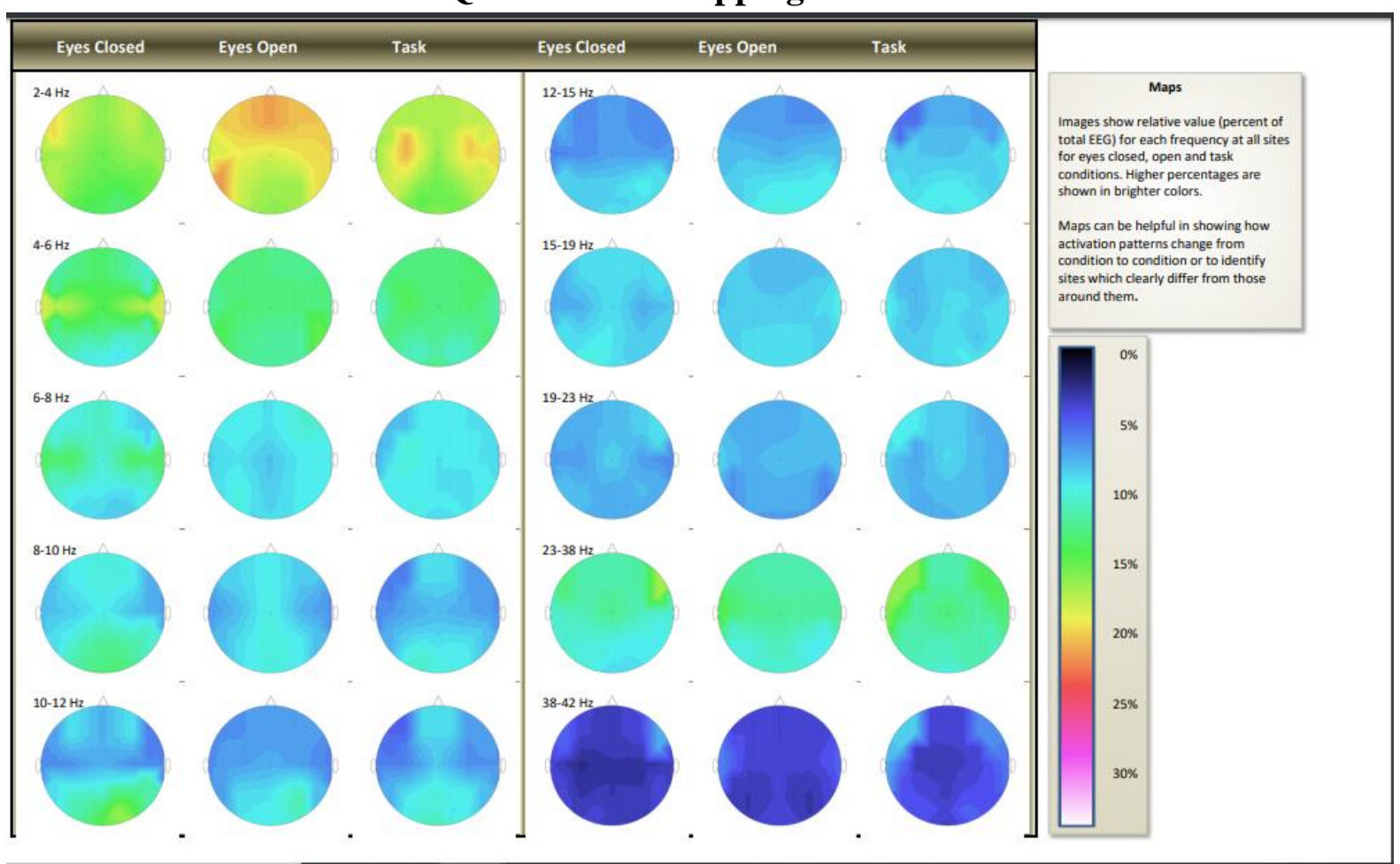

Figure 3.1 Pre-intervention QEEG brain mapping assessment result

In Figure 3.1, it shows the brainwave activity of K's brain under 3 various states before receiving neurofeedback training (i.e., eyes closed, eyes open and on-task). It indicated the dominance of slow bandwidth brainwaves, namely delta, theta and alpha brainwaves 
(according to the activation level shown in the colour spectrum) on the frontal brain and cingulate gyrus associated regions of $\mathrm{K}$ during eyes open state. Besides, the somatosensory association cortex also displayed dominance of the delta brainwave. Meanwhile, both medial and lateral sides of left and right hemispheres, namely the temporal lobes as well as parietal lobes were highly dominated by hi-beta brainwaves under all 3 different states. In addition to that, the gamma brainwave was found to be dominant, specifically at both the left and right frontal brain regions. In simpler words, this infers that the mentioned brain regions were highly active during most of the time despite when $\mathrm{K}$ was asleep or awake. In terms of the 10-20 international system, the affected brain regions were known as F7, Fz, F8 for the frontal parts, $\mathrm{Cz}, \mathrm{C} 3, \mathrm{C} 4$ for the somatosensory association cortex, and T3, T4, P4 for the temporal and parietal regions.

\subsection{The Post-Intervention of QEEG Brain Mapping Assessment}

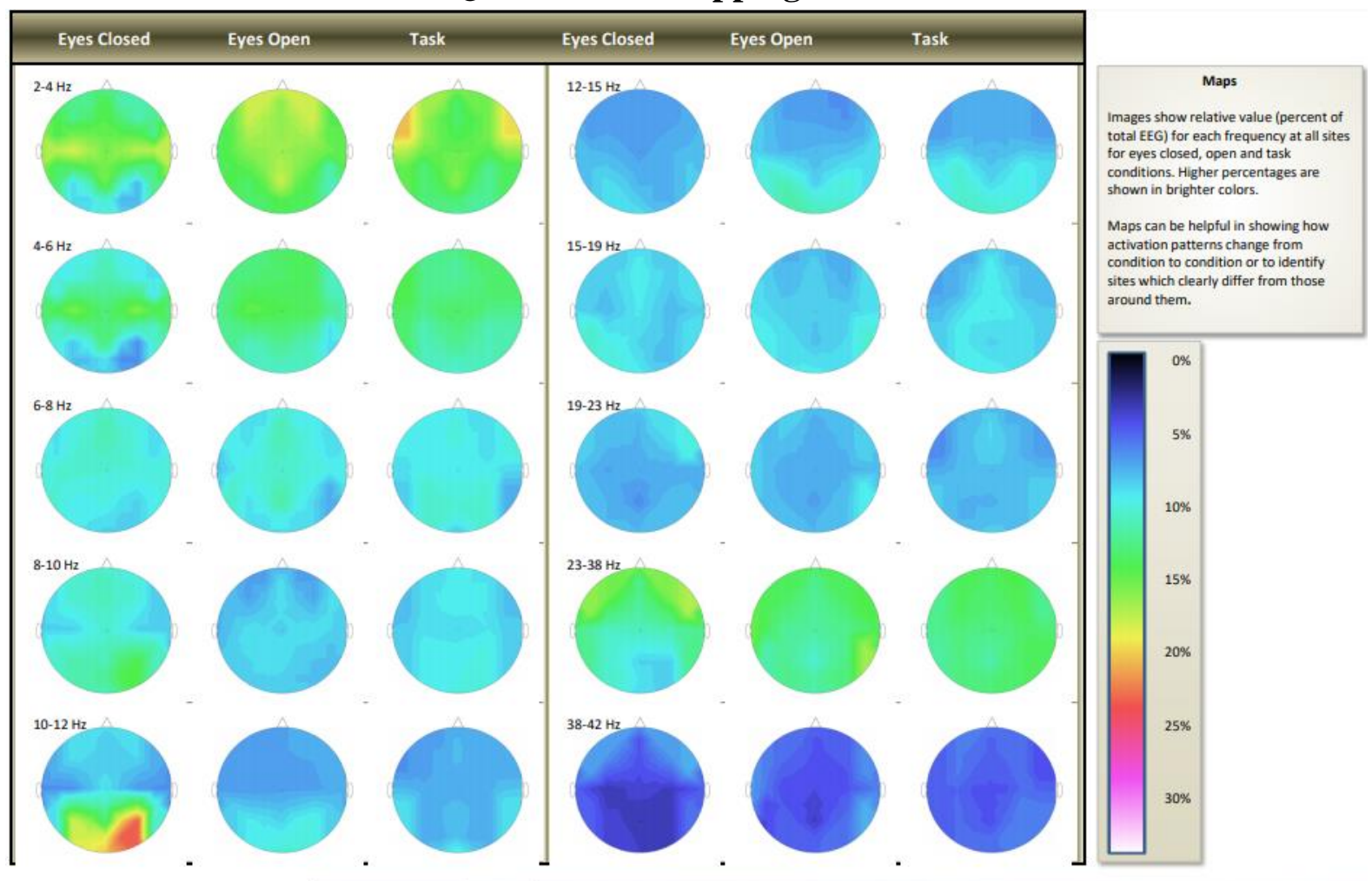

Figure 3.2 Post-intervention of QEEG brain mapping assessment result

Figure 3.2 shows the result of QEEG brain mapping assessment of $K$ after having a total of 74 sessions of clinical neurofeedback training. As compared to Figure 3.1, the dominance of slow bandwidth brainwave (i.e., delta and alpha) has reduced, particularly during the eyes open state in the frontal regions and the cingulate gyrus associated brain regions. Meanwhile, the neural activation of the beta brainwave, also known as the working brainwave, was found to be increased in the frontal regions during the eyes opened and on-task state. Besides, the gamma brainwave which is associated with anxiety and tension was found to reduce significantly at 
both left and right frontal brain parts. In addition, the activation of hi-beta brainwaves was found to display slight changes across the 3 different states after a few sessions of training.

The aforementioned outcomes of intervention indicated that the developed neurofeedback training protocols were effective and feasible in handling $\mathrm{K}$ 's psychological issue and promoting his cognitive rehabilitation process. Meanwhile, the protocol of expressive arts application in using the New Mind Card as the facilitator were also found to be effective in enhancing the treatment efficiency.

\subsection{DISCUSSIONS}

After we went through the intervention, the dominance of delta brainwave in K's frontal lobe has significantly reduced. As such, it increases the functioning level of the frontal lobe regions. To enable the frontal lobe regions to function efficiently, delta brainwaves need to be low when one's in an awake state and an on-task state (Marzbani et al., 2016). As a result, frontal lobe regions' related functions such as high-ordering cognitive processing, mood regulation, motor function, and language skills were found to improve in K (Heatherton, 2011; King et al., 2012; Zoefel et al., 2011). In the self-report diary log, K's father has reported that he has shown a significant decrease in obsessive behaviours and thus, a further reduction of his anxious feelings. This has concurred with the previous literature as frontal parts of the brain are responsible for emotional and behavioural control (Ball et al., 2013; Lu et al., 2017; Sherin \& Nemeroff, 2011). Henceforth, the neurofeedback training protocol which developed to reduce the delta brainwave at the frontal lobe regions (i.e., rewarding SMR brainwave at F7 and F8 sites) of $\mathrm{K}$ was found to be effective in promoting his self-control and emotion regulation.

In the meantime, the aforementioned improvements of $\mathrm{K}$ also could be attributed to the neurofeedback training protocol of rewarding SMR brainwave at $\mathrm{Fz}$ and $\mathrm{Cz}$, which are the cingulate gyrus associated brain regions that helps for the self-regulation, emotional control and executive functioning (Stevens et al., 2011). King et al. (2012) emphasised the increase in executive functioning could enhance one's impulse inhibition and emotion control. Therefore, K's regressive behaviours were reduced as improvement in executive functioning enabled him to control his thoughts and impulsivity. This result was parallel with previous study which revealed that neurofeedback is equally effective as cognitive rehabilitation (computer training software) in improving executive functions and reinforcing the self-regulation effect (Fashami, Akbari \& Khanzadeh, 2020).

Furthermore, the present finding showed the neurofeedback training protocol of rewarding beta brainwave at C3 was helpful in alleviating the ruminative tendency and anxiety of the client. As stated by Gao et al. (2016), low beta brainwave is associated with a mindfulness state. The reduced rumination of $\mathrm{K}$ could be attributed to the decreased gamma brainwave at F7 and F8 that is associated with anxiety and tension as well. Moon and the team (Choi, Jung, Lee \& Choi, 
2018) highlighted that the gamma brainwave at frontal brain parts was significantly associated with the anxiety and rumination tendency that was rooted from past traumatic experiences. The current finding has postulated that the neurofeedback training protocol of rewarding beta brainwave at the C3 site, and the protocol of rewarding SMR brainwave at F7 and F8 sites (particularly dominated by the hi-beta and gamma brainwave), was feasible in promoting the mindfulness skills which helps in reducing the ruminative thoughts.

Apart from that, the anxiety of $\mathrm{K}$ was improved by implementing the neurofeedback training protocol of rewarding alpha brainwaves at the right frontal lobe regions. This was in line with the previous findings which have found that the increase of alpha brainwave was effective in reducing anxious feelings with long lasting effects (Moradi et al, 2011). For this, the implemented neurofeedback protocol which rewarding alpha at the frontal lobe was believed to help K for his sleep onset issue. Previous studies have highlighted that increased neural activation level on the frontal lobe region can help to reduce the ruminative and anxious feelings, in turn promoting relaxation and ease one's sleep onset issue (Heatherton, 2011; Ramsawh et al. 2019). Therefore, the increase of alpha brainwaves can promote general calmness and emotion regulation which beneficial for K's sleep quality as well.

In addition, the implementation of the deep-calming neurofeedback training protocol which rewards the delta brainwave at $\mathrm{T} 4$ and $\mathrm{P} 4$ sites during eyes close state, was found to be helpful in tackling the reported sleep issue of K. Prior empirical studies showed that an increase of delta brainwaves during eyes-close state at P4 to be associated with deep sleep or energy restoring sleep (Arns \& Kenemans., 2014). In addition, good sleep quality influences one's emotion regulation (Finan et al., 2015). Therefore, increase of the delta brainwave ratio, which was mainly attributed to the decrement of the fast bandwidth brainwave during the eye closed state, was found to be associated with the reported improvement of K's sleep quality in his father's logbook.

Meanwhile, the use of the New Mind Card has enabled the client to express his internal dialogue metaphorically and it was found helpful in motivating $\mathrm{K}$ to commit in the neurofeedback training process according to the caregiver's feedback. It was believed that the use of expressive art therapy fosters a safe therapeutic environment and in turn, it may help in activating the social engagement system (Furman \& Boeve, 2018). Polyvagal theory reveals that the social engagement system will be activated when the ventral vagal complex of the autonomic nervous system is in the optimal state (Ogden et al., 2020). It provides a higher degree of flexibility in communication, a better ready engagement with the environment, greater stress tolerance and a better bodily self-regulation. The social engagement system also helps individuals to calm down the amygdala (limbic system), regulates brainstem nuclei and also fosters a better self-control for the cortex part of the brain indirectly (Ogden et al., 2020). 
The use of the expressive art application also provides a chance to listen to the metaphors of the client about the state of their physical/psychological interface (Weinberger \& Stoycheva, 2019). This process was aligned with the narrative storytelling process which is the social relationship experience that helps to foster a better therapeutic relationship in brain injury rehabilitation work (D'Cruz, Douglas \& Serry, 2020). The use of expressive art application in the current study also blends into different stages of neurofeedback training procedure accordance to the training location of the brain. This integrative concept is actually parallel with the historical movement of expressive art therapy as it is commonly blended into various approaches such as humanistic, cognitive, developmental and psychoanalytic (Malchiodi, 2020).

\subsection{CONCLUSION}

The primary aim of this case report study was to develop the helpful process by integrating neurofeedback training with expressive art therapy protocol. The findings indicated that the developed trauma-informed protocols for neurofeedback training and expressive art application were helpful in fostering a better therapeutic effect. This study has bridged the gap in the limited literature of lacking in the theoretical consensus, by documenting how the trauma-informed neurofeedback and trauma-informed expressive art therapy are helpful in improving the brain function, particularly for a brain injury case. Innovation of the New Mind Card also contributed to the trauma-informed practice field by providing a new method for participants in recognising the trauma impacts on their psychological well-being and give a voice for their unconscious process in expressing themselves. Future research is encouraged to adopt the developed trauma-informed neurofeedback training and expressive art therapy protocols of this present report to further consolidate effectiveness.

\subsection{ACKNOWLEDGEMENT}

I would to specially convey my sincere gratitude to those who have assisted me in producing this case report, especially the interns from New Mind Brain Health Centre, they are Mr. Lau Chen Xi, Ms. Kerk Qian Wei, and Ms Kong Wei Qin. They have provided a great assistance in collating the information about case history and caregiver's logbook. Meanwhile, they were very enthusiastic in brainstorming about the writing structure of the case report during the initial phase. It is worth to be noted that the finalized writing structure that applied in this case report was based on their idea. In a nutshell, I felt very grateful to have the chance to publish this case report and I hope my paper can contribute a new insight to the field in providing hope to those who are in need. 
International Journal of Innovation, Creativity and Change. www.ijicc.net

Volume 15, Issue 4, 2021

\section{REFERENCES}

Ali, J., Viczko, J., \& Smart, C. (2020). Efficacy of Neurofeedback Interventions for Cognitive Rehabilitation Following Brain Injury: Systematic Review and Recommendations for Future Research. In Journal of the International Neuropsychological Society, 26(1), 3146. doi:10.1017/S1355617719001061

Arns, M., \& Kenemans, J. L. (2014). Neurofeedback in ADHD and insomnia: Vigilance stabilization through sleep spindles and circadian networks. In Neuroscience and Biobehavioral Reviews, 44, 183-194. Retrieved from https://doi.org/10.1016/j.neubiorev.2012.10.006

Ball, T. M., Ramsawh, H. J., Campbell-Sills, L., Paulus, M. P., \& Stein, M. B. (2013). Prefrontal dysfunction during emotion regulation in generalized anxiety and panic disorders. Psychological medicine, 43(7), 1475-1486. doi:10.1017/S0033291712002383

Chiang, M., Reid-Varley, W. B., \& Fan, X. (2019). Creative Art Therapy for Mental Illness. Psychiatry Research, 275, 129-136

D’Cruz, K., Douglas, J., Serry, T. (2020). Sharing stories of lived experience: A qualitative analysis of the intersection of experiences between storytellers with acquired brain injury and storytelling facilitators. British Journal of Occupational Therapy, 83(9), 576-584. https://doi.org/10.1177/0308022619898085

Fashami, N. A., Akbari, B., Khanzadeh, A. A. H. (2020). Comparison of the effectiveness of cognitive rehabilitation and Neurofeedback on improving the executive functions in children with Dyslexia. Journal of Child Mental Health, 7(2), 294-311. http://dx.doi.org/10.29252/jcmh.7.2.25

Fenster, R. J., Lebois, L., Ressler, K. J., \& Suh, J. (2018). Brain circuit dysfunction in posttraumatic disorder: from mouse to man. In Nature reviews. Neuroscience, 19(9), 531551. doi: 10.1038/s41583-018-0039-7

Finan, P. H., Quartana, P. J., \& Smith, M. T. (2015). The effects of sleep continuity disruption on positive mood and sleep architecture in healthy adults. In Sleep, 38(11), 1735-1742. doi: $10.5665 /$ sleep. 5154

Furman, L., Boeve, H. (2018). Itinerant art therapy: An educational model for community outreach therapeutic service. The Arts in Psychotherapy, 57, 65-71. https://doi.org/10.1016/j.aip.2017.09.003

Gao, J., Fan, J., Wu, B. W., Zhang, Z., Chang, C., Hung, Y. S., ... Sik, H. H. (2016). Entrainment of chaotic activities in brain and heart during MBSR mindfulness training. In Neuroscience Letters, 616, 218-223. https://doi.org/10.1016/j.neulet.2016.01.001

Gapen, M., van der Kolk, B. A., Hamlin, E., Hirshberg, L., Suvak, M., \& Spinazzola, J. (2016). A pilot study of neurofeedback for chronic PTSD. In Applied Pyschophysical Biofeedback, 41, 251-261. doi: 10.1007/s10484-015-9326-5

Gomes, J. S., Ducos, D. V., Akiba, H., \& Dias, A. M. (2016). A neurofeedback protocol to improve mild anxiety and sleep quality. In Brazilian Journal of Psychiatry, 38(3), 264265. Retrieved from http://dx.doi.org/10.1590/1516-4446-2015-1811 
International Journal of Innovation, Creativity and Change. www.ijicc.net

Volume 15, Issue 4, 2021

Goodwin, G. M. (2015). The overlap between anxiety, depression, and obsessive-compulsive disorder. In Dialogues in Clinical Neuroscience, 17(3), 249-260.

Grieder, M., Homan, P., Federspiel, A., Kiefer, C., \& Hasler, G. (2020). Increased Anxiety After Stimulation of the Right Inferior Parietal Lobe and the Left Orbitofrontal Cortex. In Frontiers in Psychiatry, 11, 375. doi: 10.3389/fpsyt.2020.00375

Grodd, W., Kumar, V. J., Schuz, A., Lindig, T., \& Scheffler, K. (2020). The anterior and medial thalamic nuclei and the human limbic system: tracing the structural connectivity using diffusion-weighted imaging. In Scientific Reports, 10, 10957. https://doi.org/10.1038/s41598-020-67770-4

Heatherton T. F. (2011). Neuroscience of self and self-regulation. In Annual review of psychology, 62, 363-390. doi:10.1146/annurev.psych.121208.131616

Jensen, M. P., Grierson, C., Tracy-Smith, V., Bacigalupi, S. C., \& Othmer, S. (2008). Neurofeedback treatment for pain associated with complex regional pain syndrome Type I. In Journal of Neurotherapy, 11(1), 45-53. doi:10.1300/J184v11n01_04

Jo, H. J., Kenney-Jung, D. L., Balzekas, I., Welker, K. M., Jones, D. T., Croarkin, P. E., Benarroch, E. E., Worrell, G. A. (2019). Relationship Between Seizure Frequency and Functional Abnormalities in Limbic Network of Medial Temporal Lobe Epilepsy. In Frontiers in Neurology, 10, 488. doi: 10.3389/fneur.2019.00488

Kadosh, K. C., Staunton, G. (2019). A systematic review of the psychological factors that influence neurofeedback learning outcomes. In NeuroImage, 185, 545-555. https://doi.org/10.1016/j.neuroimage.2018.10.021

King, K. M., Fleming, C. B., Monahan, K. C., \& Catalano, R. F. (2011). Changes in self-control problems and attention problems during middle school predict alcohol, tobacco, and marijuana use during high school. In Psychology of addictive behaviors : journal of the Society of Psychologists in Addictive Behaviors, 25(1), 69-79. doi:10.1037/a0021958

Legarda, S. B., McMahon, D., Othmer, S., \& Othmer, S. (2011). Clinical neurofeedback: case studies, proposed mechanism, and applications for pediatric neurology practice. In Journal of Child Neurology, 26(8), 1041-1051. doi: 10.1177/0883073811405052

Linardakis, A., \& Pardell, R. I. (2018). The integration of quantitative EEG (QEEG) brainmapping with rTMS therapy. In Brain Stimulation, 11(6), e11. Retrieved from https://doi.org/10.1016/j.brs.2018.07.009

Lu, S., Pan, F., Gao, W., Wei, Z., Wang, D., Hu, S., ... Li, L. (2017). Neural correlates of childhood trauma with executive function in young healthy adults. In Oncotarget, 8(45), 79843-79853. doi: 10.18632/oncotarget.20051

Malchiodi, C. A. (2020). Trauma and Expressive Arts Therapy: Brain, Body, and Imagination in the Healing Process. The Guilford Press.

Marzbani, H., Marateb, H. R., \& Mansourian, M. (2016). Marzbani. In Basic and clinical neuroscience, 7(2), 143-158. doi:10.15412/J.BCN.03070208

Moon, S. Y., Choi, Y. B., Jung, H. K., Lee, Y. I., \& Choi, S. H. (2018). Increased frontal gamma and posterior delta powers as potential neurophysiological correlates 
International Journal of Innovation, Creativity and Change. www.ijicc.net

Volume 15, Issue 4, 2021

differentiating posttraumatic stress disorder from anxiety disorders. In Psychiatry investigation, 15(11), 1087-1093. doi:10.30773/pi.2018.09.30

Moradi, A., Pouladi, F., Pishva, N., Rezaei, B., Torshabi, M., \& Mehrjerdi, Z. A. (2011). Treatment of anxiety disorder with neurofeedback: Case Study. In Procedia- Social and Behavioral Sciences, 30, 103-107. Retrieved from https://doi.org/10.1016/j.sbspro.2011.10.021

National Institutes of Health (NIH). (2007). The use of complementary and alternative medicine in the United States. In National Center for Complementary and Integrative Health. Retrieved from https://nccih.nih.gov/research/statistics/2007/camsurvey_fs1.htm

National Organization for Rare Disorders (NORD). (2019). Wilson Disease. In Rare Disease Database. Retrieved from https://rarediseases.org/rare-diseases/wilson-disease/

Nolen-Hoeksema, S. (2000). The role of rumination in depressive disorders and mixed anxiety/depressive symptoms. Journal of Abnormal Psychology, 109(3), 504-511. Retrieved from https://doi.org/10.1037/0021-843X.109.3.504

Ochsner, K. N., Silvers, J. A., \& Buhle, J. T. (2012). Functional imaging studies of emotion regulation: A synthetic review and evolving model of the cognitive control of emotion. In Annals of the New York Academy of Sciences, 1251 (1), E1-E24.. doi: 10.1111/j.17496632.2012.06751.x

Ogden, P., Minton, K., Pain, C. (2020). Trauma and the body: A sensorimotor approach to Psychotherapy. Tantor Media Inc.

Orndorff-Plunkett, F., Singh, F., Aragon, O. R., \& Pineda, J. A. (2017). Assessing the Effectiveness of Neurofeedback Training in the Context of Clinical and Social Neuroscience. In Brain Sciences, 7(8), 95. doi: 10.3390/brainsci7080095

Putman, J. A., Othmer, S. F., Othmer, S., \& Pollock, V. E. (2005). TOVA results following inter-hemispheric bipolar EEG training. In Journal of Neurotherapy, 9(1), 37-52. doi: 10.1300/J184v09n01_04

Ramsawh, H. J., Stein, M. B., Belik, S. L., Jacobi, F., \& Sareen, J. (2009). Relationship of anxiety disorders, sleep quality, and functional impairment in a community sample. In Journal of Psychiatric Research, 43(10), 926-933. Retrieved from https://doi.org/10.1016/j.jpsychires.2009.01.009

Riley S. (2001). Art therapy with adolescents. The Western journal of medicine, 175(1), 5457. doi:10.1136/ewjm.175.1.54

Rogala, J., Jurewicz, K., Paluch, K., Kublik, E., Cetnarski, R., \& Wrobel, A. (2016). The Do's and Don'ts of Neurofeedback Training: A Review of the Controlled Studies Using Healthy Adults. In Frontiers in Human Neuroscience, 10, 301. doi:10.3389/fnhum.2016.00301

Sherin, J. E., \& Nemeroff, C. B. (2011). Post-traumatic stress disorder: the neurological impact of psychological trauma. In Dialogues in Clinical Neuroscience, 13(3), 263-278

Spinazzola, J., Cook, A., Ford, J., Lanktree, C., Blaustein, M., Cloitre, M., DeRosa, R., Hubbard, R., Kagan, R., Liautaud, J., Mallah, K., Olafson, E., van der Kolk, B. (2005). 
International Journal of Innovation, Creativity and Change. www.ijicc.net

Volume 15, Issue 4, 2021

Complex Trauma in Children and Adolescents. In Psychiatric Annals, 35, 390-398. doi: 10.3928/00485713-20050501-05.

Stevens, F. L., Hurley, R. A., Taber, K. H., Hurley, R. A., Hayman, L. A., \& Taber, K. H. (2011). Anterior cingulate cortex: Unique role in cognition and emotion. In The Journal of Neuropsychiatry and Clinical Neurosciences, 2(2), 121-125. Retrieved from https://neuro.psychiatryonline.org/doi/full/10.1176/jnp.23.2.jnp121

Strehl, U. (2014) What learning theories can teach us in designing neurofeedback treatments. In Frontiers in Human Neuroscience. 8:894. doi: 10.3389/fnhum.2014.00894

Torres, A. R., Fontenelle, L. F., Shavitt, R. G., Ferrao, Y. A., Rosario, M. C., Storch, E. A., \& Miguel, e. C. (2016). Comorbidity variation in patients with obsessive-compulsive disorder according to symptom dimensions: Results from a large multicentre clinical sample. In Journal of Affective Disorders, 190, 508-516. Retrieved from https://doi.org/10.1016/j.jad.2015.10.051

Wang, D., Zhu, T., Chen, J., Lu, Y., Zhou, C., Chang, Y. (2020). Acute aerobic exercise ameliorates cravings and inhibitory control in Heroin addicts: Evidence from eventrelated potentials and frequency bands. Frontiers in Psychology, 11, 2496. https://doi.org/10.3389/fpsyg.2020.561590

Wang, J. R., \& Hsieh, S. (2013). Neurofeedback training improves attention and working memory performance. In Clinical Neurophysiology, 124 (12), 2406-2420. Retrieved from http://dx.doi.org/10.1016/j.clinph.2013.05.020

Weinberger, J., Stoycheva, V. (2019). The Unconscious: Theory, Research, and Clinical Implications. The Guilford Press.

Zoefel, B., Huster, R. J, \& Hermann, C. S. (2011). Neurofeedback training of the upper alpha frequency band in EEG improves cognitive performance. In NeuroImage 54 (2), 1427 1431. Retrieved from https://doi.org/10.1016/j.neuroimage.2010.08.078 
International Journal of Innovation, Creativity and Change. www.ijicc.net Volume 15, Issue 4, 2021

\section{Appendix: Trauma-informed New Mind Card}

Example of New Mind Card

